\title{
EFEITO DA UTILIZAÇÃO DE ACARICIDAS EM CITROS, SOBRE A POPULAÇÃO DE Brevipalpus phoenicis (GEIJSKES, 1939) E ÁCAROS PREDADORES (PHYTOSEIIDAE)
}

\author{
M.E. SATO; A. RAGA; L.C. CERÁVOLO; A.C. CEZÁRIO; A.C. ROSSI \\ Instituto Biológico, C.P. 7119. CEP: 01064-970 - São Paulo, SP.
}

RESUMO: Fol conduxido experimento com o objettvo de estudar o efelto de alguns acartcldas sobre Brevipalpus phoenicis (Geijskes,1939) (Acari:Tenuipalpidae) e ácaros predadores (Phytoselldae), em citros. Os produtos testados e as dosagens em $\mathrm{g}$ i.a. $/ 100 \mathrm{l}$ de água foram: fenpyroximate $(5,0)$, acrinathrin $(0,5)$; hexythiaxox $(1,5)$; amitrax $(40,0)$ e enxofre $(225,0)$. Os acaricidas fenpyroximate, acrinathrin e hexythiazox foram eficientes contra $B$. phoenicis até 127 dias após a aplicacão, enquanto que, o enrofre e o amitraz apresentaram bom controle até 92 e 58 dias após o tratamento, respectivamente. O hexythiazox foi praticamente inócuo aos ácaros predadores mas os demais acaricidas foram significativamente prejudiciais a estes ácaros. Os produtos enxofre, fenpyroximate e acrinathrin indưiram reduçōes na populaçāo de fitoseifdeos até 58 dias, porém o amitraz apresentou reduções até 92 dias após a pulverização.

Descritores: Brevipalpus phoenicis, controle químico, Phytoselidae, citros

\section{EFFECT OF ACARICIDE UTILIZATION IN CITRUS ON THE POPULATION OF Brevipalpus phoenicis (GEIJSKES, 1939) AND PREDATORY MITES (PHYTOSEIIDAE)}

ABSTRACT: The experiment was carried out in order to study the effects of some acaricides on Brevipalpus phoenicis (Geijskes, 1939) (Acari,Tenuipalpidae) and predatory mites (Phytoselidae) in citrus. The products tested and dosages in g AI 100 l of water were: fenpyroximate (5.0), acrinathrin (0.5), hexythisaox (1.5), amitraz (40.0) and sulphur (225.0). The acaricides fenpyroximate, acrinathrin and herythiazor were eficient against $B$. phoenicis up to 127 days from the application date, whereas sujphur and amitraz presented good control up to 92 days and 58 days after treatment, respectively. Hexythiazox was fairty innocuous to predatory mites but, the remaining acaricides caused significant mortality to these mites. The pesticides sulphur, fenpyrorimate and acrinathrin induced reductions of the population of phytoseid mites up to 58 days, but amitraz showed reductions up to 92 days after application.

Key words: Brevipalpus phoenicis, chemical control, Phytoselidae, citrus

\section{INTRODUÇÃO}

A expansão da citricultura brasileira nos últimos anos trouxe muitos problemas para o sistema de produção, particularmente no tocante a pragas e doenças (GRAVENA, 1990). Neste contexto, o ácaro Brevipalpus phoenicis (Geijskes, 1939) (Acari: Tenuipalpidae) tem representado a praga mais im. portante para a citricultura paulista, devido aos seus danos e o elevado custo de seu controle (SALVO FILHO \& SALVO, 1991).

Este ácaro, considerado transmissor da leprose dos citros (MUSUMECI \& ROSSETTI, 1963; CHIAVEGATO et al. 1982), afeta significativamente a produção, devido à diminuição no peso $e$ queda prematura de frutos, além da perda de folhas e seca de ramos, em pomares infestados.

Assim sendo, toma-se de relevante importância a implementação do manejo ecológico da praga, no pomar cítrico, com ênfase na preservação dos inimigos naturais presentes. Entre os predadores, destacam-se os ácaros da familia Phytoseiidae, que exercem controle biológico sobre os ácaros fitófagos cha- ves (ferrugem e leprose) em citros (GRAVENA, 1990).

MORAES et al. (1986) mencionaram as espécies Euseius cirrifolius Denmark \& Muma,1970; Euseius concordis (Chant, 1959) Iphiseiodes quadripilis (Banks, 1904) e Iphiseiodes zuluagai Denmark \& Muma 1972, entre outras, presentes em nossos pomares cítricos.

RAGA et al. (1993) estudaram a distribuição dos ácaros predadores (Phytoseiidae) na planta cítrica, observando maior ocorrência destes ácaros em folhas localizadas nos terços médio e inferior da copa, principalmente naquelas com teias de insetos (Ordem Psocoptera). Foram encontrados 3,25 vezes mais ácaros por unidade de folha do que de fruto.

Diversos trabalhos de controle químico do ácaro da leprose tem sido realizados no Estado de São Paulo, nos últimos anos (CALAFIORI et al. , 1986; MOTTA et al., 1987; RAGA et al., 1990; SATO et al., 1991 b; SCARPELLINI et al.,1991; CHIAVEGATO, 1993; VENDRAMINI et al., 1993). Também tem sido conduzidos diferentes trabalhos 
visando a observação do efeito de produtos químicos sobre os ácaros predadores e outros inimigos naturais presentes em pomar cítrico (SCARPELLINI \& NAKANO, 1989; SATO et al., 1991a ; GRAVENA , 1992; SATO et al., 1992; YAMAMOTO et al., 1992; SILVEIRA et al., 1993).

KOMATSU \& NAKANO (1988) verificaram que o enxofre (80\%) na dosagem de $80 \mathrm{~g} / 100 l$ de calda, não afetava a população de $E$. concordis, em condições de laboratório.

MORSE et al. (1987) estudando a toxicidade residual de vários acaricidas a Euseius stipulatus Athias- Henriot,1960, na Califómia, verificaram que - fenbutatin oxido e o avermectin mais óleo foram praticamente inócuos ao ácaro, enquanto que, os produtos cihexatin e amitraz apresentaram mortalidade inicial alta (acima de $80 \%$ ), porém com rápido declínio.

$O$ objetivo do presente experimento foi observar o efeito de alguns acaricidas sobre o ácaro $B$. phoenicis e ácaros predadores da família Phytoseiidae, em pomar cítrico.

\section{MATERIAL E MÉTODOS}

O experimento foi realizado na Estação Experimental do Instituto Biológico, em Presidente Prudente, SP, em pomar de laranjeiras, c.v. Pera Rio, com 10 anos de idade, de espaçamento de $7 \mathrm{~m}$ entre ruas e $5 \mathrm{~m}$ entre plantas.

$O$ delineamento estatístico adotado foi inteiramente casualizado com 6 tratamentos e 4 repe- tições. Os tratamentos e as dosagens em g i.a./100 litros de água foram: fenpyroximate (Kendo $50 \mathrm{SC}$ ) a 5,0; acrinathrin (Rufast $50 \mathrm{SC}$ ) a 0,5; hexythiazox (Savey 500 PM) a 1,5; amitraz (Amitraz $200 \mathrm{CE}$ Defensa) a 40,0; enxofre (Uniflow 750 SC) a 225,0 e testemunha.

Cada parcela foi constituída de 12 plantas (3 ruas de 4 plantas), sendo avaliadas apenas as 2 plantas internas da rua central. A pulverização foi realizada no dia 06/05/93, com emprego de pistola, gastando-se em média $20 l$ de calda por planta.

Em cada planta útil foram amostrados ao acaso 10 frutos e 10 folhas. $O$ critério utilizado na avaliação do ácaro da leprose nos frutos, foi baseado na metodologia adotada por RAGA et al. (1990), e constituiu-se na contagem do número de ácaros presentes na área correspondente ao deslocamento da lupa (aumento de 10 vezes e com campo visual de $1 \mathrm{~cm}^{2}$ ), desde o pendúnculo até o extremo oposto do fruto. Nas folhas, as observações foram realizadas ao longo da nervura, nas faces dorsal e ventral.

No caso dos fitoseifdeos, a avaliação era realizada em toda superfície das folhas e frutos coletados. Durante as avaliações foram retiradas amostras destes ácaros predadores, para posterior identificação em laboratório.

Uma avaliaçăo prévia foi conduzida em 14/ $04 / 93$ e as demais avaliaçzes foram realizadas aos $13,28,58,92$ e 127 dias após a aplicação.

$O$ número de ácaros da leprose $\mathrm{e}$ fitoseídeos foi transformado em $\sqrt{X+0,5}$ e analisados através dos testes F e Tukey a $5 \%$ de probabilidade.

TABELA 1- Efeito de acaricidas sobre o ácaro da leprose, Brevipalpus phoenicis em citros; número médio de ácaros por parcela (20 frutos) $\quad\left(n^{\circ}\right)$; porcentagem de redução populacional (\%Red) nas diversas avaliações realizadas. Presidente Prudente, SP, abril a setembro de 1993.

\begin{tabular}{|c|c|c|c|c|c|c|c|c|c|c|c|}
\hline \multirow[t]{3}{*}{ Tratamentos } & \multirow{3}{*}{$\frac{\text { Prévia }}{\mathrm{N}^{\circ}}$} & \multicolumn{10}{|c|}{ dias após a aplicação } \\
\hline & & \multicolumn{2}{|c|}{13} & \multicolumn{2}{|c|}{28} & \multicolumn{2}{|c|}{58} & \multicolumn{2}{|c|}{92} & \multicolumn{2}{|c|}{127} \\
\hline & & $\mathbf{N}^{\circ}$ & $\%$ Red & $\mathrm{N}^{\circ}$ & $\%$ Red & $\mathrm{N}^{\circ}$ & $\%$ Red & $\mathbf{N}^{\circ}$ & $\%$ Red & $N^{\circ}$ & $\% \operatorname{Red}$ \\
\hline $\begin{array}{l}\text { fenpyroximate } \\
\text { acrinathrin } \\
\text { hexythiazox } \\
\text { amitraz } \\
\text { enxofre } \\
\text { testemunha }\end{array}$ & $\begin{array}{c}27,0 \text { a } \\
16,8 \text { a } \\
20,5 \text { a } \\
19,8 \text { a } \\
28,3 \text { a } \\
22,5 \text { a }\end{array}$ & $\begin{array}{r}1,8 \mathrm{a} \\
1,8 \mathrm{a} \\
6,3 \mathrm{a} \\
2,5 \mathrm{a} \\
3,3 \mathrm{a} \\
19,5 \mathrm{~b}\end{array}$ & $\begin{array}{l}91,0 \\
91,0 \\
68,0 \\
87,2 \\
83,3 \\
-\end{array}$ & $\begin{array}{c}0,0 \mathrm{a} \\
0,3 \mathrm{a} \\
1,0 \mathrm{a} \\
1,0 \mathrm{a} \\
1,3 \mathrm{a} \\
14,0 \mathrm{~b}\end{array}$ & $\begin{array}{r}100,0 \\
98,2 \\
92,9 \\
92,9 \\
91,1 \\
-\end{array}$ & $\begin{array}{r}0,0 \mathrm{a} \\
0,3 \mathrm{a} \\
1,0 \mathrm{a} \\
4,3 \mathrm{a} \\
1,8 \mathrm{a} \\
21,5 \mathrm{~b}\end{array}$ & $\begin{array}{r}100,0 \\
98,8 \\
95,4 \\
80,2 \\
91,9 \\
-\end{array}$ & $\begin{array}{r}0,3 \mathrm{a} \\
0,0 \mathrm{a} \\
0,3 \mathrm{a} \\
14,5 \mathrm{c} \\
4,5 \mathrm{~b} \\
24,8 \mathrm{c}\end{array}$ & $\begin{array}{r}99,0 \\
100,0 \\
99,0 \\
41,4 \\
81,81 \\
-\end{array}$ & $\begin{array}{l}1,5 \mathrm{a} \\
1,3 \mathrm{a} \\
1,5 \mathrm{a} \\
19,8 \mathrm{~b} \\
10,8 \mathrm{~b} \\
17,0 \mathrm{~b}\end{array}$ & $\begin{array}{r}91,2 \\
92,7 \\
91,2 \\
0,0 \\
36,8 \\
-\end{array}$ \\
\hline C.V. $(\%)$ & 17,60 & 35,93 & & 23,61 & & 49,67 & & 24,26 & & 29,05 & \\
\hline
\end{tabular}

Calculada pela formula de Abbott

Sci. agric., Piracicaba, 52(2):282-286, mai./ago. 1995 


\section{RESULTADOS E DISCUSSÃo}

Fazendo-se as observações em folhas e frutos, verificou-se que a maioria dos ácaros da leprose $(96,1 \%)$ estavam presentes nos frutos. Por outro lado, $82,0 \%$ dos ácaros predadores foram encontrados $\mathrm{em}$ folhas, concordando com RAGA et al. (1993) que observaram predominância de fitoseiideos em folhas.

$O$ efeito dos acaricidas, sobre a população do ácaro da leprose, pode ser observado na TABELA 1. Os produtos fenpyroximate e acrinathrin mostraram-se efetivos no controle de $B$. phoenicis até 127 dias após a aplicą̧ão, com controle superior a $91,0 \%$, em todas as avaliações.

O hexythiazox também foi efetivo, porém na primeira avaliação, a porcentagem de redução populacional foi de apenas $68 \%$. A partir da segunda contagem, a eficiência atingiu niveis satisfatórios, com reduçzes acima de $91 \%$.

Bons resultados no controle da praga utilizando o hexythiazox tamberm foram obtidos por MOTTA et al., 1987; SCARPELLINI et al., $1991 \mathrm{e}$ SATO et al., 1992.

Os acaricidas amitraz e enxofre apresentaram-se inferiores aos produtos anteriormente mencionados, nas avaliações realizadas aos 92 e 127 dias após a aplicação. $O$ amitraz mostrou-se eficiente até 58 dias após a pulverização, enquanto que, o enxofre apresentou bom controle até 92 dias do tratamento.

VENDRAMINI et al. (1993), trabalhando com a mesma formulação do enxofre (SC), também observaram boa eficiência do produto contra o ácaro da leprose, no município de Piracicaba, SP.

Quanto aos fitoseiídeos, foram encontrados ácaros principalmente das espécies $I$. zuluagai, $E$. citrifolius e $E$. concordis. As duas últimas espécies ocorreram em maior número nos meses finais do experimento (Agosto, Setembro).

$O$ efeito dos tratamentos sobre os ácaros predadores, pode ser visualizado na TABELA 2. O hexythiazox não se mostrou significativamente prejudicial aos fitoseiideos, com reduções inferiores a $22 \%$. Estes resultados concordam com KOMATSU \& NAKANO (1988), que testaram o produto para $E$. concordis, em laboratório. Porém, YAMAMOTO et al. (1992) constataram toxicidade moderada do defensivo a Euseius sp., em pomar cítrico de Jaboticabal, SP.

Os demais acaricidas causaram reduções significativas na população de ácaros predadores no campo. Aos 13 dias após a aplicação, a porcentagem de redução foi acima de $80 \%$, para estes produtos.

$O$ enxofre apresentou-se significativamente prejudicial à população de predadores até 58 dias após a aplicação. $O$ índice de redução variou de 74,3 a $80,3 \%$, neste período. Resultados semelhantes já haviam sido obtidos em experimento realizado anteriormente na região (SATO et al., 1992). Embora o produto tenha afetado a populaçăo de fitoseiídeos no campo, KOMATSU \& NAKANO (1988) verificaram que o enxofre era praticamente inócuo a $E$. concordis, em laboratório.

TABELA 2- Efeito de acaricidas sobre os ácaros predadores (Phytoseiidae) em citros; número médio de ácaros por parcela (20 folhas) $\left(n^{\circ}\right)$; porcentagem de redução populacional (\%Red) nas diversas avaliações realizadas. Presidente Prudente, SP, abril a setembro de 1993.

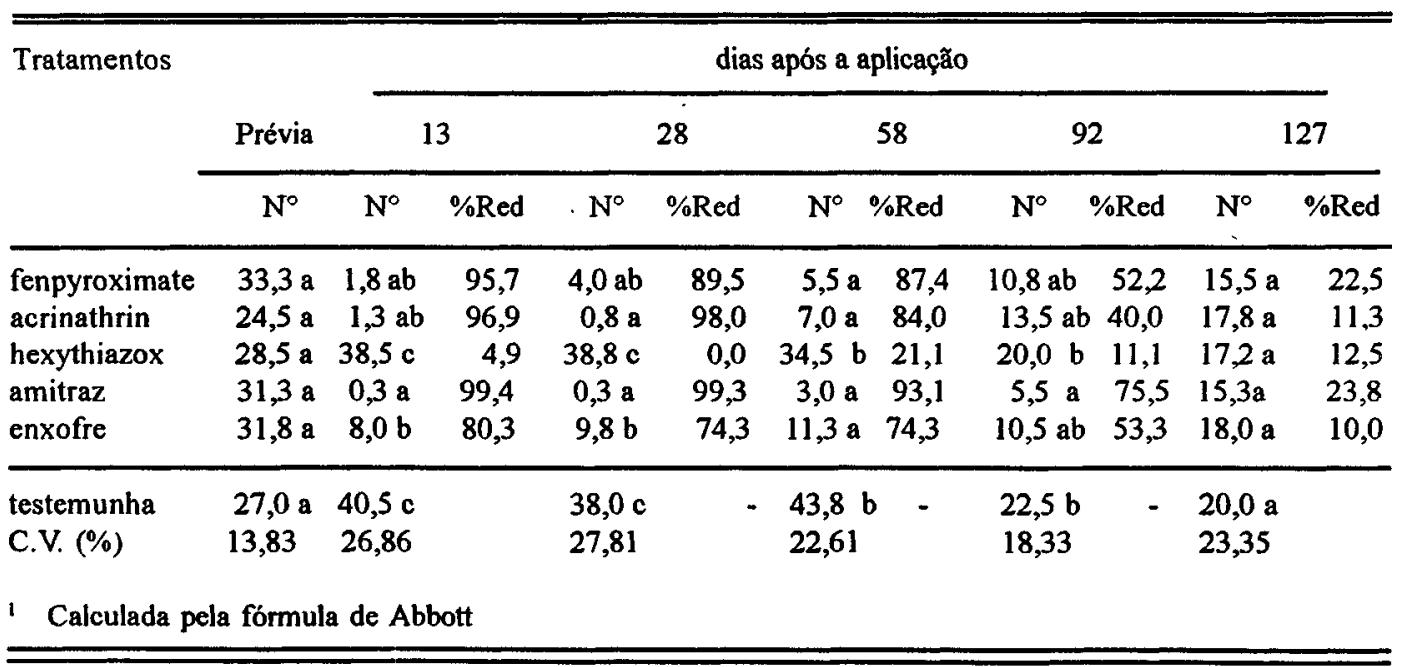


Os acaricidas fenpyroximate e acrinathrin mostraram um comportamento semelhante de toxicidade, aos fitoseiídeos no campo. Inicialmente, apresentaram reduções acima de $95 \%$, ocorrendo gradual retorno da população destes predadores, ao longo do experimento. Da mesma forma que no tratamento com enxofre, aos 92 dias após a aplicação, não se observou mais diferença significativa entre a população de fitoseiídeos nestes tratamentos e na testemunha.

O produto amitraz também foi bastante prejuóicial aos ácaros predadores, com redução populacional de $99,4 \%$ aos 13 dias após a pulverização. Mesmo aos 92 dias após o tratamento a população de fotoseiídeos ainda permanecia baixa $(75,5 \%$ de redução) apresentando-se significativamente inferior a da testemunha. Apenas aos 127 dias, esta população tornou-se semelhante a das plantas não tratadas. A alta mortalidade inicial causada por amitraz em fitoseiídeos, também foi observada por MORSE et al. (1987), na Califórnia.

Não foi observado nenhum sintoma de fitoxicidade devido ao uso destes acaricidas no pomar cítrico.

Estes resultados servem de subsídio para a escolha adequada de acaricidas, para o controle da praga, considerando-se a grande importância da manutenção de inimigos naturais, como os ácaros predadores, num programa de Manejo Integrado de Pragas em citros.

\section{CONCLUSŌES}

- Os acaricidas fenpyroximate, acrinathrin e hexythiazox foram eficientes contra o ácaro da leprose até 127 dias após a aplicação, enquanto que, o enxofre e o amitraz mostraram controle satisfatório até 92 e 58 dias, respectivamente.

- O hexythiazox foi praticamente inócuo aos ácaros predadores (Phytoseiidae).

- Os acaricidas enxofre, fenpyroximate e acrinathrin apresentaram reduções significativas na população de fitoseiídeos até 58 dias após a aplicação (até 92 dias para amitraz).

\section{REFERÊNCLAS BIBLIOGRÁFICAS}

CALAFIORI, M.H.; ALVAREZ, E.J.; FERRAZ, J.C.; GIORGETTI. C.L.; PALLINI FILHO, A; MENDES, E.; COTTAS, M.P. Controle do ácaro da leprose Brevipalpus phoenicis (Geijskes, 1939) em laranjeiras, Citrus spp.em duas regiðes do Estado de São Paulo. Ecossistema, Espirito Santo do Pinhal, v.11, p. 53-60, 1986
CHIAVEGATO, L.G.; MISCHAN M.M.; SILVA, M.de A. Prejuizos e transmissibilidade de sintomas de leprose pelo ácaro Brevipalpus phoenicis (Geijskes, 1939) Sayed, 1946 (Acari, Tenuipalpidae) em citros. Científica, Jaboticabal, v.10, n. 2, p. 265-271, 1982.

CHIAVEGATO, L.G. Controle químico do ácaro da leprose Brevipalpus phoenicis (Geijskes, 1939) (Acari: Tenuipalpidade) em condições de laboratório. In: CONGRESSO BRASILEIRO DE ENTOMOLOGIA, 14., Piracicaba, 1993. Resumos... Piracicaba: Sociedade Entomológica do Brasil, 1993.p. 715.

GRAVENA, S. Manejo ecológico de pragas no pomar cítrico. Laranja, Cordeirópolis, v.11, n. 1, p. 205-225, 1990.

GRAVENA, S. MIP Citros: avanços e inovações na citricultura brasileira. Laranja, Cordeirópolis, v.13, n. 2, p. $635-691,1992$. .

KOMATSU,S.S. \& NAKANO, O. Estudos visando o manejo do ácaro da leprose em citros através do ácaro predador Euseius concordis. Laranja, Cordeirópolis, v.9, n. 1, p.125-146, 1988.

MORAES, G.J. de; Mc MURTRY, J.A; DENMARK, H.A. A catalog of the mite family Phytoseiidae: references to taxonomy, synonymy, distribution and habitat. Brasilia: EMBRAPA - DDT, 1986. 353p.

MORSE, J.G.; BELLOWS Jr.,T.S.; GASTON, L.K.; IWATA, Y. Residual toxicity of acaricides to three beneficial species on California citrus. Journal of Economic Entomology, College Park, v. 80, n. 4, p.953-960, 1987.

MOTTA, R.; SILVA, J.M.; SUGAHARA, C.A; RAIZER, A.J.; KATO, W.Y. MARICONI, F.A.M. Pulverização de novos acaricidas no combate ao ácaro da leprose Brevipalpus phoenicis (Geijskes, 1939) (Acari: Tenuipalpidae) em citros. Anais da Escola Superior de Agricultura "Luiz de Queiroz",Piracicaba, v. 44, n. 1, p. 811-824, 1987.

MUSUMECI, M.R. \& ROSSETTI, V. Transmissão dos sintomas da leprose dos citros pelo ácaro Brevipalpus phoenicis. Ciência e Cultura, São Paulo, v. 15, n. 3, p. $228,1963$.

RAGA, A. SATO, M.E.; CERÁVOLO, L.C.; ROSSI, A.C.; SCARPELLNI J.R.. Ação de acaricidas sobre o ácaro da leprose Brevipalpus phoenicis (Geijskes, 1939) em pomar cítrico de Presidente Prudente, SP. Ecossistema, Espírito Santo do Pinhal, v. 15, p.98-103, 1990.

RAGA,A; SATO, M.E.; CERÁVOLO, L.C.; ROSSI, A.C. Distribuição de ácaros predadores (Phytoseiidae) em laranjeira (Citrus sinensis L. Osbeck) In: REUNIÃO ANUAL DO INSTITUTO BIOLÓGICO, 6., São Paulo, 1993. Resumos... São Paulo: Instituto Biológico, 1993.p. 42. 
SALVO FILHO, A. de \& SALVO, S. de. Tratamentos fitossanitários dos citros. 1991. Laranja, Cordeirópolis, v. 12, n. 2, p.289-313, 1991.

SATO, M.E.; RAGA, A; CERÁVOLO, L.C.; ROSSI, A.C. Efeito de acaricidas sobre Brevipalpus phoenicis (Geijskes, 1939) e à fauna de artrópodos, em citros. In: REUNIÃO ANUAL DO INSTITUTO BIOLÓGICO, 4., São Paulo, 1991 a. Resumos... São Paulo: Instituto Biológico, 1991a, p. 24.

SATO, M.E.; CERÁVOLO, L.C.; ROSSI, A.C.; SCARPELLINI, J.R.; POTENZA, M.R. Controle químico do ácaro da leprose Brevpalpus phoenicıs (Gejjskes, 1939) (Acari: Tenuipalpidae) em pomar cítrico de Presidente Prudente. Arquivos do Instituto Biológico, São Paulo, v.58, n. 1/2, p.25-28, 1991 b.

SATO,M.E.; RAGA,A.; CERÁVOLO, L.C.; ROSSI, AC.; CEZÁRIO,A.C. Efeito de acaricidas sobre Brevipalpus phoenicis (Geijskes, 1939) (Acari:Tenuipalpidae) écaros predadores (familia: Phytoseiidae) em citros. Revista Brasileira de Fruticultura, Cruz das Almas, v.14, p.87-93, 1992.

SCARPELLINI, J.R. \& NAKANO, O. Seletividade do ácaro predador Euseius spp (Acari, Phytoseiidae) a alguns acaricidas na cultura dos citros. In: CONGRESSO BRASILEIRO DE ENTOMOLOGIA, 12., Belo Horizonte, 1989. Resumos... Belo Horizonte: Sociedade Entomológica do Brasil, 1989. p. 423.
SCARPELLINI, J.R.; SATO, M.E.; TAKEMATSU, A.P. \& RAGA, A. Efeito de acaricidas sobre o ácaro da leprose Brevipalpus phoenicis (Geijskes, 1939) no município de Bebedouro, SP. Revista de Agricultura, Piracicaba, v.66, p.183-192, 1991.

SILVEIRA, D.A.; BUZOLIN, P.R.; PAIVA, P.E.B.; YAMAMOTO, P.T.; GRAVENA, S. Toxicidade do acaricida-inseticida flufenoxuron (Cascade $100 \mathrm{CE}$ ) sobre Euserus citrifolius (Denmark \& Muma) (Acari: Phytoseiidae) em citros. In: CONGRESSO BRASILEIRO DE ENTOMOLOGIA, 14., Piracicaba, 1993. Resumos... Piracicaba: Sociedade Entomológica do Brasil, 1993. p. 601 .

VENDRAMINI, J.M.B., NIVOLONI, R.F; NAKANO, O. Ensaio visando o controle do ácaro da leprose (Brevpalpus phoenicis, Geijskes, 1939) dos citros. In: CONGRESSO BRASILEIRO DE ENTOMOLOGIA, 14., Piracicaba, 1993. Resumos... Piracicaba: Sociedade Entomológica do Brasil, 1993. p.440.

YAMAMOTO, P.T.; PINTO, A.de S.; PAIVA, P.E.B.; GRAVENA, S. Seletividade de agrotóxicos aos inimigos naturais de pragas dos citros. Laranja, Cordeirópolis, v.13, n. 2, p.709-755, 1992.

Recebido para publicação em 21.06 .94

Aceito para publicação em 24.10 .94 